\title{
Quantum noise limits of a semiconductor laser with dispersive optical feedback
}

Yaakov Shevy, Hua Deng, Sven Mahnkopf, John Kitching, Amnon Yariv

Yaakov Shevy, Hua Deng, Sven Mahnkopf, John Kitching, Amnon Yariv, "Quantum noise limits of a semiconductor laser with dispersive optical feedback," Proc. SPIE 3611, Laser Resonators II, (24 May 1999); doi: $10.1117 / 12.349261$

SPIE Event: Optoelectronics '99 - Integrated Optoelectronic Devices, 1999, San Jose, CA, United States 


\title{
QUANTUM NOISE LIMITS OF A SEMICONDUCTOR LASER WITH DISPERSIVE OPTICAL FEEDBACK.
}

\author{
Yaakov Shevy, Hua Deng, and Sven Mahnkopf. \\ Department of Physics, University of Miami, P.O Box 248046, Coral Gables, Fl 33146. \\ John Kitching and Amnon Yariv \\ Department of Applied Physics, California Institute of Technology, Pasadena, CA 91125.
}

\begin{abstract}
$\underline{\text { Abstract }}$
The quantum noise limits of a semiconductor laser with dispersive loss are examined theoretically and experimentally. Using optical feedback from Cs vapor as a dispersive loss element we demonstrate almost 6 orders of magnitude reduction in the linewidth, to a $44 \mathrm{~Hz}$ level, and $1.9 \mathrm{~dB}$ amplitude noise squeezing below the standard quantum noise limit.
\end{abstract}

Key words: Semiconductor laser, linewidth, FM noise, AM noise.

\section{Introduction}

In the last decade the semiconductor laser has become one of the most important optical device and is now being used for myriad applications. To many of these, low laser noise is of utmost importance. For example, low noise lasers are needed for communications networks, microwave links and precision spectroscopy. However, the semiconductor laser frequency noise is quite substantial compared to other laser systems. Whereas in other lasers the main noise source is low frequency technical noise, the quantum noise of the small cavity semiconductor laser is many orders of magnitude larger and is on the order of a few MHz. This is because the laser linewidth, given by the Schawlow-Townes formula, is inversely proportional to the cavity volume.

Moreover, the semiconductor laser linewidth is even larger than the Schawlow-Townes limit by a factor of $\left(1+\alpha^{2}\right)$, where $\alpha$, the amplitude-phase coupling parameter, is in the range of 3-6 [1-2]. The amplitude-phase coupling mechanism, which is unique to semiconductor lasers, generates excess phase noise due to the changes in the refractive index induced by amplitude fluctuations. Hence, the combined effects of the small cavity and the amplitude to phase coupling lead to a broad, quantum mechanical in origin, linewidth of a few tens of $\mathrm{MHz}$. Furthermore, the frequency stability of the semiconductor laser is also adversely affected by current and temperature fluctuations.

In our research we have taken a new look at the basic noise limits of the semiconductor laser. We discovered that ironically the phase to amplitude coupling mechanism could be exploited to quench the laser noise by many orders of magnitude below the Schawlow-Townes limit. This can be achieved by coupling the laser to a frequency dependent loss mechanism [4]. A semiclassical theoretical analysis indicates that the inclusion of a frequency dependent loss (or gain) process characterize by a "slope"(as a function of frequency), $\mathrm{C}$, in the laser cavity can give rise to a dramatic reduction of the laser linewidth given by the following formula:

$$
\Delta v=\Delta v_{\text {S.T }} \frac{\left(1+\alpha^{2}\right)}{(1+\mathrm{C} \alpha)^{2}}
$$

As can be clearly seen, for $\mathrm{C} \alpha \gg 1$ the laser linewidth becomes smaller than the Schawlow-Townes limit by a factor of $1 / \mathrm{C}^{2}$. We demonstrated experimentally such a reduction of a semiconductor laser linewidth by using a dispersive optical feedback from atomic Cs vapor [5]. In agreement with a detailed theoretical analysis using the "dispersive loss" concept [7,8] which predicted values of $\mathrm{C} \sim 1000$, we show that the frequency noise power was indeed reduced by almost six orders of magnitude below the quantum noise level.

The semiclassical analysis of the amplitude noise in the presence of dispersive loss suggested another startling result, this noise can be reduced by a factor $\left(1+C^{2}\right) /(1+C \alpha)^{2}$. As can be seen, in the limit of large $C$, the amplitude noise could be below the Standard Quantum Limit (SQL) or the "shot noise" by a factor of $\alpha^{2}$. To test this prediction, however, a full 
quantum mechanical analysis was required. We have recently initiated this analysis and found that such a reduction is possible close to threshold but is more limited at high pump rates. We were able to demonstrate these phenomena experimentally and achieve amplitude noise squeezing by $1.9 \mathrm{~dB}$ below the SQL at room temperature.

\section{Theoretical results and background:}

We first review the general semiclassical model, then analyze the situation in which an external frequency-dependent element provides a weak optical feedback to the semiconductor laser, and finally discuss the full quantum model. To demonstrate the effects of a frequency-dependent loss we use the Van der Pol laser oscillator equations driven by spontaneous emission. The dispersive loss mechanism is represented [4] by a frequency-dependent photon lifetime, $\tau_{\mathrm{p}}^{-1}=\tau_{\mathrm{p} 0}^{-1}+2 \mathrm{C} \dot{\varphi}$. To include the attendant frequency-dependent index change $C$ becomes a complex quantity, $C=C_{r}+i C_{i}$, where $C_{r}$ represents a frequency-dependent loss and $C_{i}$ a frequency-dependent index change. The modified Van der Pol equations are, therefore, given by[5]:

$$
\begin{aligned}
& \dot{\delta}+\omega_{1} \delta+A_{0} C_{\mathrm{r}} \dot{\varphi}=\Delta_{\mathrm{i}} / 2 \omega_{\mathrm{m}} \\
& \mathrm{A}_{0}\left(1+\mathrm{C}_{\mathrm{i}}\right) \dot{\varphi}-\alpha \omega_{1} \delta=-\Delta_{\mathrm{r}} / 2 \omega_{\mathrm{m}} \quad \text { Eq. } 1
\end{aligned}
$$

Using Laplace transform methods and assuming a white Langevin driving force to represent spontaneous emission leads to a Lorentzian field spectrum with FWHM, $\Delta \mathrm{v}$, given by:

$$
\Delta v=\Delta v_{\text {S.T }} \frac{\left(1+\alpha^{2}\right)}{\left[1+\alpha C_{r}+C_{i}\right]^{2}} \equiv \Delta v_{\text {S.T }} \frac{\left(1+\alpha^{2}\right)}{Q^{2}} \quad \text { Eq. } 2
$$

Aside from elucidating the different roles of the frequency-dependent amplitude and phase, Eq. 2 enables one to find the laser linewidth in various practical situations. In particular for a system, which is composed of a semiconductor laser with an external frequency-dependent feedback, we obtain for $\mathrm{Cr}$ and $\mathrm{Ci}$

$$
\begin{aligned}
& \mathrm{C}_{\mathrm{r}}=\partial \kappa(\omega) / \partial \omega \cos \phi-\kappa(\omega) \sin \phi(\partial \phi / \partial \omega) \\
& \mathrm{C}_{\mathrm{i}}=\partial \kappa(\omega) / \partial \omega \sin \phi+\kappa(\omega) \cos \phi(\partial \phi / \partial \omega) \quad \text { Eq.3 }
\end{aligned}
$$

Where $\kappa(\omega)$ is the feedback coupling rate defined by $\kappa(\omega)=\left(1-r_{c}{ }^{2}\right) r(\omega) / r_{c} \tau_{c}, r_{c}, \tau_{c}$ are the semiconductor facet reflectivity and round trip time respectively, $\kappa(\omega)$ is the frequency-dependent external reflectivity, $\phi$ is the feedback phase, $\partial \phi / \partial \omega=\tau+\partial \phi^{\prime} / \partial \omega, \tau$ is the total external cavity round trip time, and $\partial \phi^{\prime} / \partial \omega$ is the derivative of the optical feedback phase due to the dispersion of the external reflector. Using $C_{r}$ and $C_{i}$ we obtain for the linewidth reduction factor $Q$ :

$$
\mathrm{Q} \equiv 1+\alpha \mathrm{C}_{\mathrm{r}}+\mathrm{C}_{\mathrm{i}}=1+\sqrt{1+\alpha^{2}}\left[\partial \kappa / \partial \omega \sin \left(\phi+\tan ^{-1} \alpha\right)+\kappa(\omega)\left(\tau+\partial \phi^{\prime} / \partial \omega\right) \cos \left(\phi+\tan ^{-1} \alpha\right)\right] \text { Eq.4 }
$$

The operating frequency of the laser $\omega$ is given by the frequency pulling expression:

$$
\omega=\Omega-\kappa(\omega) \sqrt{\left(1+\alpha^{2}\right)} \sin \left(\phi+\tan ^{-1} \alpha\right) \quad \text { Eq.5 }
$$

where $\Omega$ is the laser frequency without feedback. We found [5,8] that in the case of a narrow frequency-dependent feedback, $\kappa(\omega)$, the laser tends to operate near zero frequency pulling. Thus, only the cosine term in Eq.4 is important. In this case the frequency-dependent dispersion is more important than the amplitude dependence of the feedback, $\partial \kappa / \partial \omega$. We conclude, therefore, that with zero frequency pulling the maximum linewidth reduction occurs at the peak of the frequency-dependent reflectivity. Assuming a Lorentzian $\kappa(\omega)$ with FWHM of $10 \mathrm{MHz}$ and peak amplitude of $2 \mathrm{GHz}$, we estimate $\mathrm{Q} \approx 1000$. This should lead to linewidth reduction, in the case of a white frequency noise spectrum, by a factor of $10^{6}$. We note in passing that we have also performed a detailed theoretical analysis of this system using rate equations [8], which also include the carrier dynamics, and found a complete agreement with the Van der Pol analysis [5].

As mentioned above, according to the semiclassical analysis, the amplitude noise can be reduced by a factor $\left(1+C^{2}\right) /(1+\alpha C)^{2}$, where $\mathrm{C}=\mathrm{Cr} / 1+\mathrm{Ci}$. To test this prediction, however, a full quantum mechanical analysis was required. This analysis was carried out by using a quantum operator version of the rate equations[14] modified by the addition of terms accounting for the dispersive loss. These new set of equations consider the effects of vacuum fluctuations incident on the laser facet as well as the effects of pump fluctuations which become crucial at high pump rates. We have also considered the effect of an external (delayed) frequency dependent feedback.

In the low frequency limit, well below the semiconductor laser cavity bandwidth, the power spectrum of the external field amplitude fluctuations $\mathrm{P}_{\Delta r}(\Omega)$ is given by: 


$$
\mathrm{P}_{\Delta \mathrm{r}}(\Omega)=\frac{1}{2}(1-\eta)+\frac{\eta}{2}\left[\frac{1}{\mathrm{R}}+2 \mathrm{n}_{\mathrm{sp}}\left|\frac{\alpha \mathrm{C}(\Omega)-1 /\left(\mathrm{n}_{\mathrm{sp}} \mathrm{R}\right)}{1+\alpha \mathrm{C}(\Omega)}\right|^{2}+2 \mathrm{n}_{\mathrm{sp}}\left(1+\frac{1}{\mathrm{n}_{\mathrm{sp}} \mathrm{R}}\right)^{2} \frac{\mathrm{C}(\Omega)^{2}}{|1+\alpha \mathrm{C}(\Omega)|^{2}}\right]
$$

where $\eta$ is the external efficiency of the laser, $\mathrm{n}_{\mathrm{sp}}$ the spontaneous emission factor and $\mathrm{C}(\Omega)=\mathrm{C}_{\mathrm{r}}(\Omega) /\left(1+\mathrm{C}_{\mathrm{i}}(\Omega)\right)$. It has also been assumed that the feedback is not strong enough to bring the phase noise down to the shot noise so that only noise due to dipole moment and vacuum fluctuations is considered.

From these expressions a number of conclusions can be inferred. First pump noise and spontaneous emission are not affected by $\mathrm{C}$, as can be seen by the first term inside the brackets in Eq.6. The second term comes from combined contribution of the dipole and vacuum fluctuations and can be reduced with $\mathrm{C}$. The final term is the uncorrelated component of the phase noise which has been transformed to amplitude noise by the dispersive loss.

From this result one finds that at very high pump rates and with no internal losses, no significant enhancement to the squeezing can be obtained. This is because the amplitude noise due to pump fluctuations and spontaneous emission into non-lasing modes is uncorrelated with the frequency noise and thus cannot be suppressed with dispersive loss and phase-toamplitude coupling. However, at lower pump rates, the dipole moment and vacuum fluctuations dominate the noise. As in the semiclassical analysis, the frequency fluctuations are indeed correlated with these noise sources and therefore noise reduction is possible. In fact, in the limit of $R->0$ (threshold), the noise is reduced by $\left(1+C^{2}\right) /(1+\alpha C)^{2}$ which is exactly the factor which results from the semi-classical analysis. Under normal operating conditions, a 5-10dB reduction can be achieved.

\section{Experimental results:}

In our experiments a frequency-dependent optical feedback was obtained by a Doppler free Faraday effect in Cs vapor[5]. A $5 \mathrm{~cm}$ Cs vapor cell was placed inside an external cavity composed by the laser's output facet and a mirror situated $40.5 \mathrm{~cm}$ away (See figure 1). The reflection from the external mirror was extinguished with two crossed linear polarizers ( each with an extinction ratio of $1: 10^{4}$ ). The $\mathrm{Cs}$ cell was heated to $\sim 50^{0} \mathrm{C}$ and a magnetic field of $\sim 1$ Gauss was applied along the laser's propagation axis.

This arrangement produced a narrow Doppler free reflections with a FWHM of $17 \mathrm{MHz}$ at the Cs hyperfine transitions at $852 \mathrm{~nm}$. The feedback phase and amplitude were controlled with a PZT at the back mirror and a variable neutral density filter in front of it. The maximum feedback level was $-35 \mathrm{~dB}$ and was obtained at $\sim 1$ Gauss. At higher magnetic fields the narrow emission was suppressed and stronger yet broader emissions with $\sim 200 \mathrm{MHz}$ FWHM became dominant $[5,8]$.

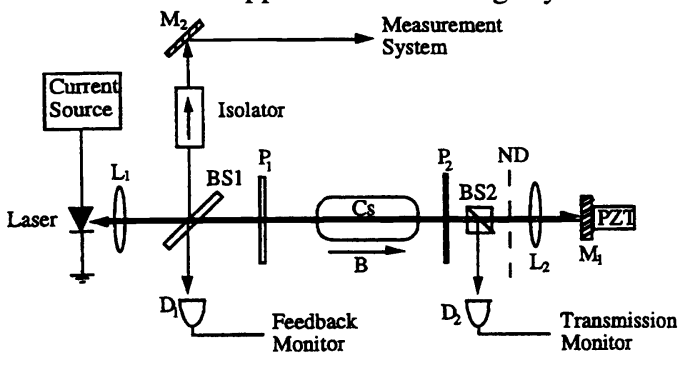

Fig. 1: The basic experimental setup for the Cs optical feedback apparatus.

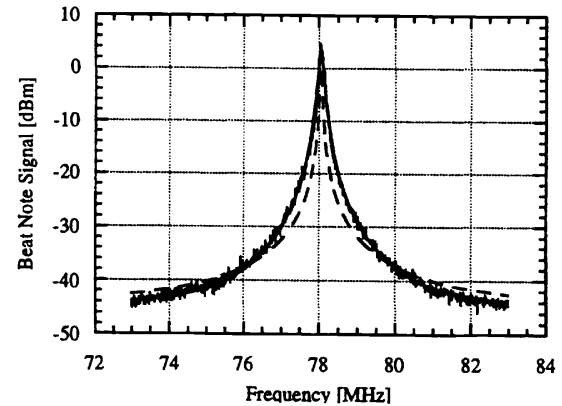

Fig. 2: The self heterodyne signal of the optically locked laser. The dashed and solid lines are a Lorentzian and a Lorentzian raised to the power of $3 / 2$.

The application of the optical feedback gave rise to a dramatic quenching of the laser linewidth. We observed a maximum reduction by a factor of 2000 , from $20 \mathrm{MHz}$ down to $10 \mathrm{kHz}$ (figure 2). However, this was well below the value expected from Eq.4 which predicts linewidth reduction by a factor of $10^{6}$. We investigated this discrepancy and found that the FM spectrum was dominated at low frequency by $1 /$ f noise component $[5,9]$. This is also evident in the self-heterodyne signal (fig. 2) which could be better fitted with a Lorentzian raised to the power 3/2. We have verified (fig. 3), as indicated by the theory, that both the white and the $1 / \mathrm{f}$ noise power were reduced by $1 / \mathrm{Q}^{2}$. In addition we were able to extract from some of the heterodyne data both the $1 / \mathrm{f}$ and the white noise contribution to the linewidth (by using a Voigt profile for the beat note signal). We found that the $1 / \mathrm{f}$ linewidth was reduced by the factor $1 / \mathrm{Q}$ while the white noise contribution to the linewidth was reduced by $1 / \mathrm{Q}^{2}$ (fig. 4). We conclude that although the linewidth was only reduced by a factor of 2000 , the white noise 
contribution to the linewidth was indeed reduced by six orders of magnitude to $\sim 10 \mathrm{~Hz}$ level .

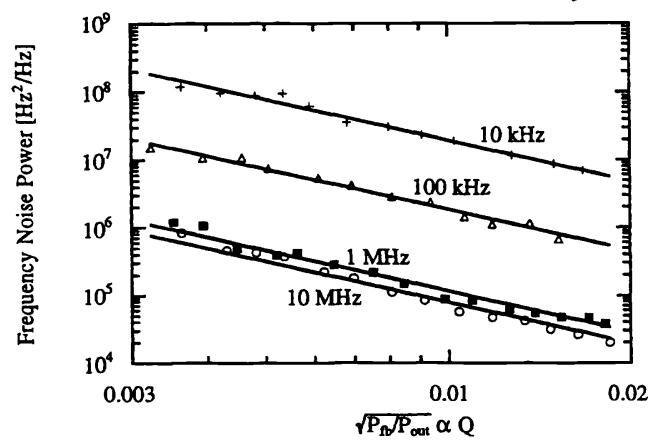

Fig. 3: The frequency noise power at various frequencies. The spectrum is uniformly reduced by $1 / \mathrm{Q}^{2}$.

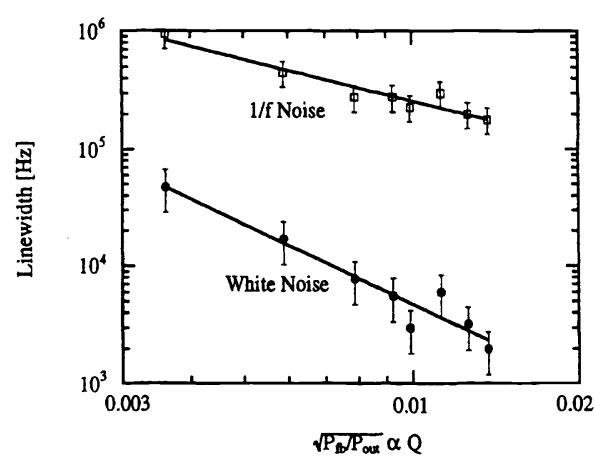

Fig. 4: The 1/f and the white noise linewidth extracted from the Voigt profile. The $1 / \mathrm{f}$ linewidth is reduced by $1 / \mathrm{Q}$, while the white noise contribution is reduced by $1 / \mathrm{Q}^{2}$.

This striking result indicates that if the $1 / \mathrm{f}$ noise component would be further eliminated one can achieve the theoretical white noise limited linewidth of few tens of $\mathrm{Hz}$. To this end we used a novel technique to reduce the $1 / \mathrm{f}$ noise[10]. In this experiment FM sidebands were generated by modulating the laser current. An error signal was obtained by utilizing the FM sideband spectroscopy signal from the Faraday resonance used for the optical feedback. This led to a reduction of the laser linewidth to $1.4 \mathrm{kHz}$ at FWHM.

Most recently, we were able to improve on this result by using an additional Cs cell for the FM sideband error signal [11]. This allowed us to optimize the optical and electronic feedback channels separately and achieve a linewidth of $44 \mathrm{~Hz}$ at FWHM. As can be seen in figure 5, the noise at most frequencies was reduced down to a level of $\approx 10 \mathrm{~Hz} / \mathrm{Hz}^{2}$, which indicates a linewidth of $30 \mathrm{~Hz}$ at FWHM. Note that compared to the free running laser the noise power at low frequencies was reduced by 9 orders of magnitude. Indeed the laser frequency stability was also improved by more than 4 orders of magnitude relative to the free running value.

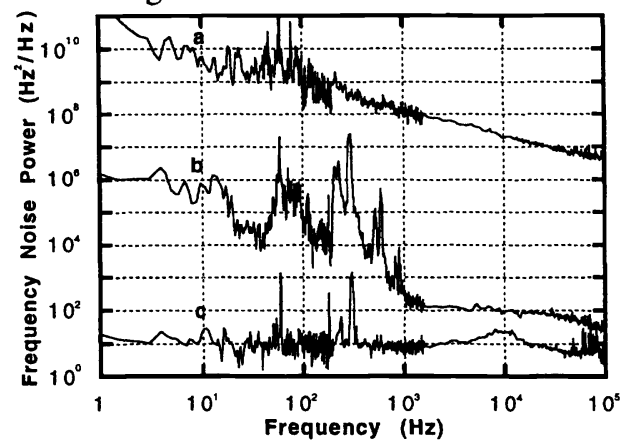

Fig. 5: The frequency noise spectrum for a free running laser (A), with a optical feedback only (B) and for both optical and electronic feedback.

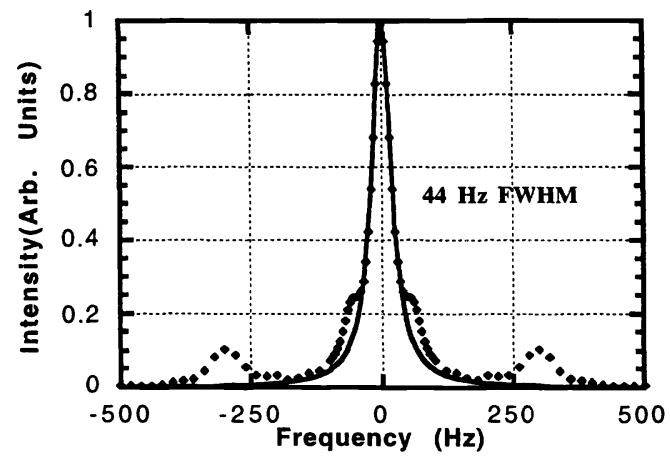

Fig. 6: The laser field spectrum of trace $C$ of Fig. 5. The solid line is a Lorentzian fit.

We should note that similar results have been achieved using optical feedback from a high finesse optical cavity. However, compared with the mechanically unstable external cavity approach [6], the Cs system offers the advantages of a stable atomic frequency standard, elimination of mode hopping, straightforward alignment and a compact device. On the other hand the external Fabry-Perot system offers broadband tunability.

In an effort to achieve amplitude noise squeezing we used essentially the same apparatus of dispersive loss from Cs vapor (figure 7). We were able to reduce the amplitude noise of a room temperature Fabry-Perot quantum well laser below the SQL by as much as $1.9 \mathrm{~dB}$ at the output facet of the laser (figure 8) [15]. We also compared the experimental data to our quantum theory of dispersive loss and obtained reasonable agreement. Our analysis revealed though the important role played by weak longitudinal sidemodes in the noise dynamics. We have developed a quantum theory of a two-mode model that better 
fits the data (figure 8). However, in some other situations we observed discrepancies even with this model, suggesting the important role played by extremely weak sidemodes in the noise dynamics and that some revisions to the basic model may still be needed.

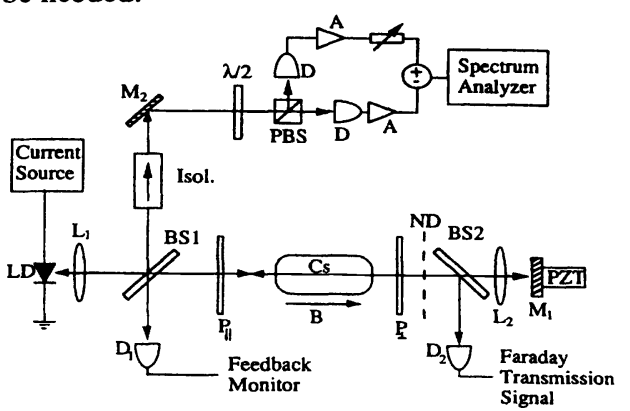

Fig. 7: The experimental apparatus for the generation and measurement of amplitude squeezed light with dispersive loss.

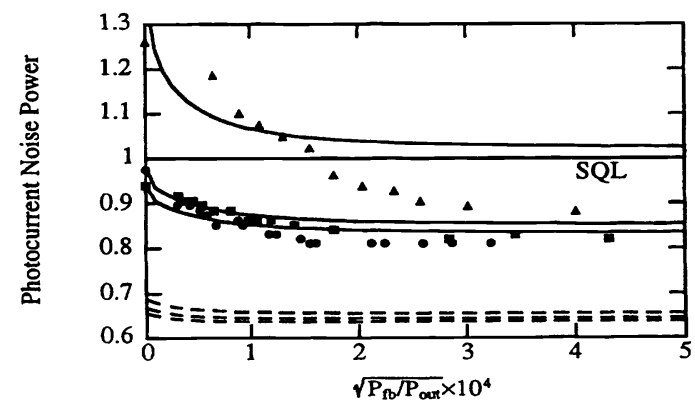

Fig. 8: The amplitude noise Vs. C at a few injection currents. The dashed and solid lines are the single and multi-mode predictions of the quantum theory.

We plan to continue the investigation of amplitude noise squeezing with dispersive loss. Most recently we studied theoretically the inclusion of a new term in the quantum noise theory $(\partial \kappa / \partial \omega)$ to account for detuned operation from the peak of the $\mathrm{Cs}$ resonance and identified new regimes with improved prospects for squeezing. In our previous analysis we took care to operate with zero frequency pulling and at the peak of the Cs resonance. As can be seen in figure 23 better results are predicted with the extended theory (which also better fit our old data) when one tunes the laser a bit away from the peak. We plan to take a second look at these effects and compare the results to the new theory [20].

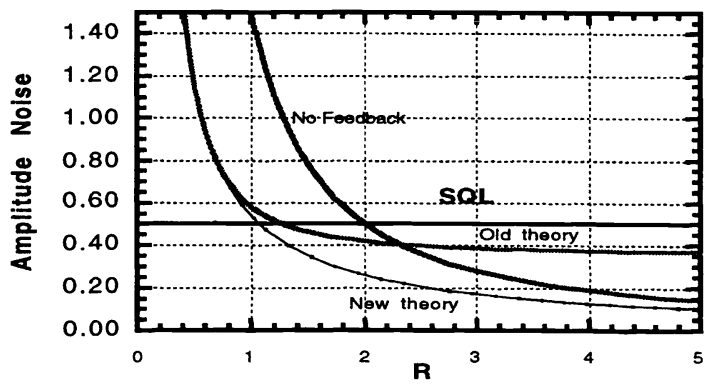

Fig. 9: The amplitude noise vs. pumping rate, comparing the new theoretical results with our previous theory[15].

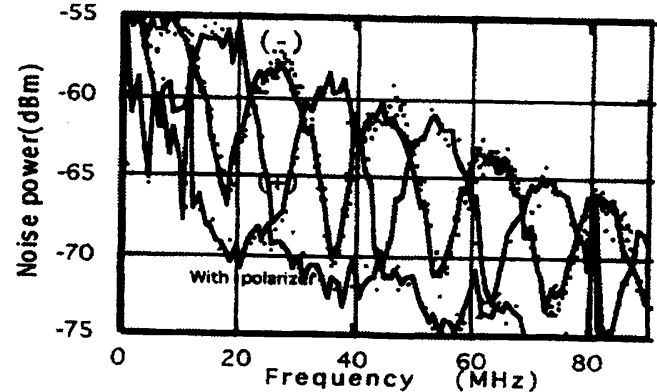

Fig. 10: The AM noise of the laser measured with a balanced Homodyne detector with/out a polarizer. A false "squeezing" is obtained $([+]$ is lower than $[-])$ due to interference with the orthogonal polarization.

Another interesting avenue is the investigation of the effects of the orthogonal polarization to the laser main mode. We found experimentally this polarization component, which typically contains $\sim 1 \%$ of the laser power, to have a profound effect on the amplitude noise squeezing. In particular, we noticed that when a polarizing beam splitter is used in the balanced homodyne detection apparatus (see figure10), large variations of the noise could be obtained by the insertion of a polarizer in front of the laser. In fact, if the measurement of the shot noise level is performed without a polarizer a higher noise level (by as much as $8 \mathrm{~dB}$ ) is obtained. This behavior disappears when a polarizer is introduced in front of the Balanced Homodyne Detector, or a polarization insensitive beam splitter is used. This suggests that larger amount of squeezing may be obtained if the full radiation field from the laser were detected with the use of a polarization insensitive beam splitter, or/and the noise level in the orthogonal polarization could be controlled. The last proposition might also be realized because there seems to be a correlation between the orthogonal noise components.

As discussed above, we also found that weak sidebands in the laser output significantly degrade the noise performance of the laser[15,17]. One of the effects of strong optical feedback is to suppress these sidemodes which may improve the 
squeezing behavior of the laser. We have observed more than $40 \mathrm{~dB}$ sidemode suppression in our previous experiments with strong feedback. We plan to investigate the amplitude noise squeezing under such conditions.

\section{Simultaneous reduction of AM and FM semiconductor laser quantum noise.}

As discussed above, optical feedback can lower both the phase and amplitude noise of semiconductor lasers. Since large reduction in the phase and amplitude noise is possible, it is pertinent to ask what are the limits of this simultaneous reduction? It is also important to verify that the Heisenberg principle as applied to the spectral uncertainty product is not violated. The quest for an answer is not purely academic because of the practical value of a semiconductor laser with a minimized spectral noise product.

The Heisenberg spectral uncertainty product is given by the following expression where $r_{0}{ }^{2}$ is the number of photons/sec:

$$
P_{\Delta \phi}(\Omega) P_{\Delta r}(\Omega) \geq \frac{1}{4 r_{0}^{2}}
$$

In a conventionally pumped laser the large amount of phase noise gives rise to a spectral uncertainty product much larger than this limit. A pump-suppressed laser with an infinite pumping rate will generate amplitude squeezed light and in this case the phase diffusion noise will increase to compensate. While the amplitude noise drops below the SQL by a factor proportional to $1 / \Omega^{2}$, the phase noise increases above the shot noise by the same factor [13].

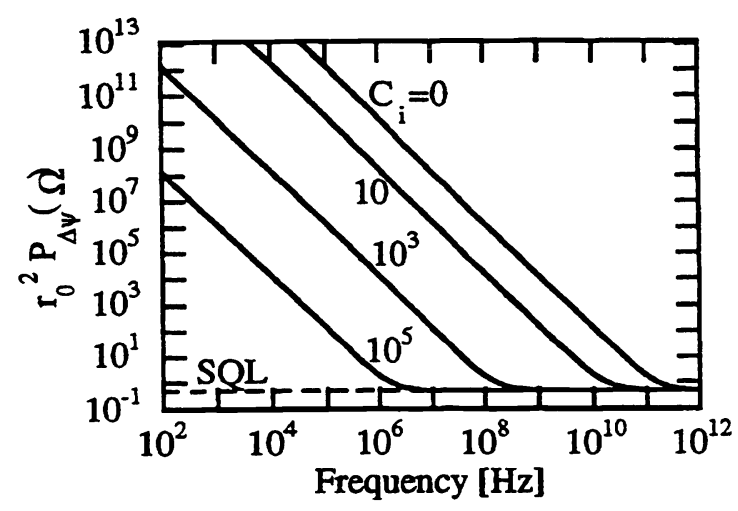

Fig. 11: The spectrum of the phase noise of a pumped suppressed semiconductor laser at a few values of $C\left(R=10^{5}\right)$.

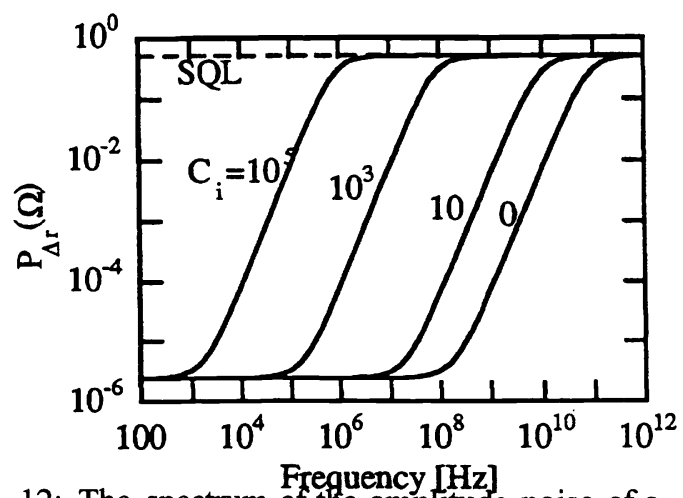

Fig. 12: The spectrum of the amplitude noise of a pumped suppressed semiconductor laser at a few values of $C\left(R=10^{5}\right)$.

The quantum analysis of the effects of optical feedback reveals that indeed the spectrum of the phase noise reduction has a significant affect on the bandwidth of the amplitude squeezing. For example, Figures 11-12 show the amplitude and phase noise as a function of frequency; in the case of very high pumping rate, pump suppression, and optical feedback, which is tailored to reduce only the phase noise $(\mathrm{Cr}=0, \mathrm{Ci}>0)$. It can be clearly seen that as the phase noise is reduced by the feedback the squeezing bandwidth is also reduced to preserve the uncertainty product. One should also note that the uncertainty product can approach unity with $\mathrm{C}=1000$ at the important $\mathrm{RF}$ range of $\Omega>1 \mathrm{MHz}$. ( Not withstanding the prohibitively high pump rate used in this figure)

We plan to continue to explore this issue both theoretically and experimentally. Clearly there is a long road ahead to achieve the minimum set by the Heisenberg uncertainty. For example, FM noise at the SQL entails a white noise linewidth on the order of a few $\mathrm{mHz}$. However, the dispersive loss technique may be a promising route to this target, since other techniques using electronic feedback to reduce the phase noise have showed an increase in the AM noise. Moreover, we have already demonstrated large values of $\mathrm{C}$, which can dramatically reduce the phase noise. Using a $17 \mathrm{MHz}$ Doppler free Faraday resonance a value of $Q \approx 1000$ has already been achieved leading to a white noise component on the order of $10 \mathrm{~Hz}$.

As can bee seen in figure 14, reduction of the phase noise by a very large factor and amplitude squeezing can be obtained simultaneously with the operational parameters that we currently obtain in a room temperature Cs cell. This theoretical plot was generated with recent addition to the quantum theory of terms due to the slope, $\partial \kappa / \partial \omega$, of the dispersive loss. 


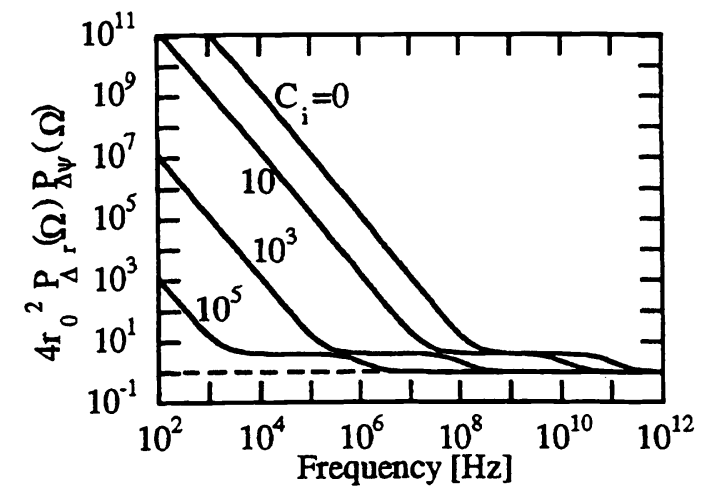

Fig. 13: The spectrum of the spectral uncertainty product of a pumped suppressed semiconductor laser at a few values of $C$ $\left(\mathrm{R}=10^{5}\right)$.

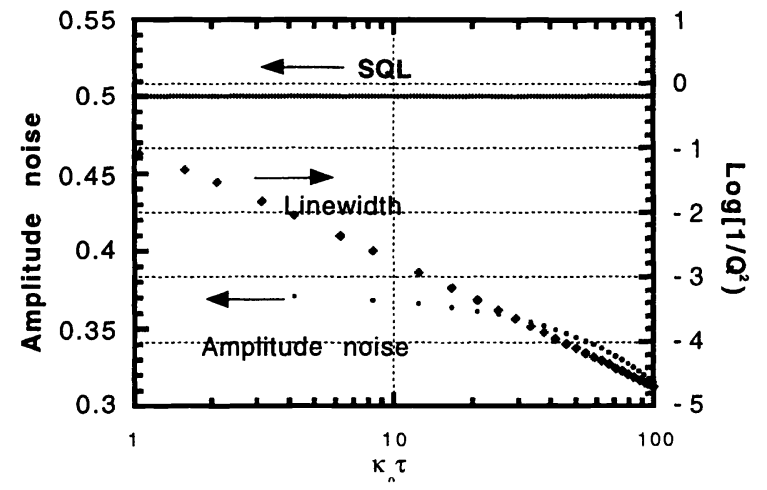

Fig. 14: Simultaneous reduction of the laser frequency and amplitude noise $(\mathrm{R}=1.5)$ as a function of the feedback coupling rate. At each point the other feedback parameters are adjusted to give maximum squeezing.

In conclusion: We have demonstrated the utility of the dispersive loss concept for the study of quantum noise reduction in semiconductor lasers. Using optical feedback as a dispersive loss element one can achieve a reduction of many orders of magnitude in the phase noise and simultaneous a few $\mathrm{dB}$ amplitude noise squeezing.

\section{Appendix 1}

We present here our solution for the power spectrum of the external laser field amplitude and phase noise. This model also includes the effects of the finite delay due to the external cavity, and the case of $\delta \kappa / \delta \omega \neq 0)$

$$
\begin{aligned}
& P_{\Delta r}(\Omega)=2\left|R_{1}\right|^{2}\left[\frac{4 k T}{q^{2} R_{s}}+(1-\beta) \frac{N_{c 0}}{\tau_{s p}}\right]+\frac{1}{2}\left|1 \frac{R_{2}\left(1+C_{i}\right)}{\sqrt{\tau_{s p}}}\right|^{2}+\frac{2 n_{s p}-1}{2 \tau_{s p}}\left|R_{2}\left(1+C_{i}\right)-2 A_{0} R_{1}\right|^{2} \\
& +\frac{1}{2 \tau_{\mathrm{sp}}}\left|\mathrm{R}_{2}\right|^{2}\left|1+\mathrm{C}_{\mathrm{i}}\right|^{2}+\frac{\mathrm{n}_{\mathrm{sp}}}{\tau_{\mathrm{sp}}}\left|\mathrm{R}_{2}\right|^{2}\left|\mathrm{C}_{\mathrm{r}}\right|^{2} \\
& \mathrm{P}_{\Delta \phi}(\Omega)=2\left|\mathrm{R}_{3}\right|^{2}\left[\frac{4 \mathrm{kT}}{\mathrm{q}^{2} \mathrm{R}_{\mathrm{s}}}+(1-\beta) \frac{\mathrm{N}_{\mathrm{c} 0}}{\tau_{\mathrm{sp}}}\right]+\frac{1}{2 \mid}\left|\frac{\sqrt{\tau_{\mathrm{pe}}}}{\mathrm{A}_{0}}-\mathrm{R}_{5} \sqrt{\frac{1}{\tau_{\mathrm{pe}}}}\right|^{2}+\frac{2 \mathrm{n}_{\mathrm{sp}}-1}{2 \tau_{\mathrm{p}}}\left|2 \mathrm{R}_{3} \mathrm{~A}_{0}+\mathrm{R}_{4}\right|^{2} \\
& +\frac{2 \mathrm{n}_{\mathrm{sp}}-1}{2 \tau_{\mathrm{p}}}\left|\mathrm{R}_{5}\right|^{2}+\frac{\left|\mathrm{R}_{4}\right|^{2}}{2 \tau_{\mathrm{p}}}+\frac{\left|\mathrm{R}_{5}\right|^{2}}{2 \tau_{\mathrm{p} 0}} \\
& \mathrm{P}_{\Delta \mathrm{r} \Delta \phi}(\Omega)=\frac{1}{\sqrt{\mathrm{P}_{\Delta \mathrm{r}}(\Omega) \mathrm{P}_{\Delta \phi}(\Omega)}}\left\{2 \mathrm{R}_{1}^{*} \mathrm{R}_{3}\left(\frac{4 \mathrm{kT}}{\mathrm{q}^{2} \mathrm{R}_{\mathrm{s}}}+(1-\beta) \frac{\mathrm{N}_{\mathrm{c} 0}}{\tau_{\mathrm{sp}}}\right)+\right. \\
& \frac{2 \mathrm{n}_{\mathrm{sp}}-1}{2 \tau_{\mathrm{sp}}}\left(4 \mathrm{R}_{1}^{*} \mathrm{R}_{3} \mathrm{~A}_{0}^{2}-2 \mathrm{~A}_{0}\left(\mathrm{R}_{1}^{*} \mathrm{R}_{4}+\mathrm{R}_{2}^{*}\left(1+\mathrm{C}_{\mathrm{i}}^{*}\right) \mathrm{R}_{3}\right)+\left(\mathrm{R}_{2}^{*} \mathrm{R}_{4}\left(1+\mathrm{C}_{\mathrm{i}}^{*}\right)-\mathrm{R}_{2}^{*} \mathrm{R}_{5} \mathrm{C}_{\mathrm{r}}^{*}\right)\right) \\
& \left.+\frac{1}{2 \tau_{\mathrm{p}}}\left(\mathrm{R}_{2}^{*} \mathrm{R}_{4}\left(1+\mathrm{C}_{\mathrm{i}}^{*}\right)-\mathrm{R}_{2}^{*} \mathrm{R}_{5} \mathrm{C}_{\mathrm{r}}^{*}\right)-\frac{1}{2 \sqrt{\tau_{\mathrm{pe}}}}\left(\mathrm{R}_{4}-\frac{\mathrm{R}_{2} \mathrm{C}_{\mathrm{r}} \sqrt{\tau_{\mathrm{pe}}}}{\mathrm{A}_{0}}\right)\right\}
\end{aligned}
$$

The first term in each expression corresponds to pump (assuming a pump suppressed operation) and spontaneous emission noise. The second term is due to vacuum fluctuation (spontaneous emission into the lasing mode). The third term is due to dipole moment fluctuations, and the fourth is due to internal losses. The last term in the amplitude noise expression is the uncorrelated phase noise that is coupled into the field amplitude due to feedback. The other quantities are defined by: $\tau_{\mathrm{st}}$ is the 
electron stimulated emission lifetime, $\tau_{\mathrm{sp}}$ is the electron spontaneous emission lifetime, $\omega$ is the lasing frequency, $\mu$ is the non-resonant index

$$
\begin{aligned}
& \mathrm{A}_{1}=-\left(\frac{1}{\tau_{\mathrm{sp}}}+\frac{1}{\tau_{\mathrm{st}}}\right)=-\frac{\left(1+\beta \mathrm{A}_{0}^{2}\right)}{\tau_{\mathrm{sp}}}, \quad \mathrm{A}_{2}=-\frac{2 \mathrm{~A}_{0}}{\tau_{\mathrm{sp}}} \quad \mathrm{A}_{3}=-\frac{\beta\left(1+\mathrm{A}_{0}^{2}\right)}{2 \mathrm{~A}_{0} \tau_{\mathrm{sp}}} \\
& \mathrm{C}_{\mathrm{i}}(\Omega)=[\partial \kappa(\omega) / \partial \omega \sin \phi+\kappa(\omega) \cos \phi(\partial \phi / \partial \omega)] \frac{1-\mathrm{e}^{-\mathrm{i} \Omega \tau}}{\mathrm{i} \Omega \tau}, \quad \mathrm{a}_{\mathrm{i}}=[\kappa(\omega) \cos \phi(\partial \phi / \partial \omega)] \frac{1-\mathrm{e}^{-\mathrm{i} \Omega \tau}}{\mathrm{i} \Omega \tau} \\
& \mathrm{C}_{\mathrm{r}}(\Omega)=[\partial \kappa(\omega) / \partial \omega \cos \phi-\kappa(\omega) \sin \phi(\partial \phi / \partial \omega)] \frac{1-\mathrm{e}^{-\mathrm{i} \Omega \tau}}{\mathrm{i} \Omega \tau}, \quad \mathrm{a}_{\mathrm{r}}=[\kappa(\omega) \sin \phi(\partial \phi / \partial \omega)] \frac{1-\mathrm{e}^{-\mathrm{i} \Omega \tau}}{\mathrm{i} \Omega \tau} \\
& R_{1}(\Omega)=\sqrt{\frac{1}{\tau_{p e}}} \frac{A_{3}}{D(\Omega)}\left[1+C_{i}+\alpha C_{r}\right], \quad R_{2}(\Omega)=\sqrt{\frac{1}{\tau_{p e}}} \frac{i \Omega-A_{1}}{D(\Omega)} \\
& R_{3}(\Omega)=\frac{1}{A_{0}} \frac{A_{3}}{D(\Omega)}\left[\alpha\left(1+a_{i}\right)-a_{r}\right] R_{4}(\Omega)=\frac{1}{A_{0}} \frac{1}{1+C_{i}+\alpha C_{r}}\left[\frac{\alpha}{i \Omega}-\frac{\left(\alpha\left(1+a_{i}\right)-a_{r}\right)\left(i \Omega-A_{1}\right)}{D(\Omega)}\left(1+C_{i}\right)\right] \\
& \begin{array}{l}
R_{5}(\Omega)=\frac{1}{A_{0}} \frac{1}{1+C_{i}+\alpha C_{r}}\left[\frac{\alpha}{i \Omega}+\frac{\left(\alpha\left(1+C_{i}\right)-C_{r}\right)\left(i \Omega-A_{1}\right)}{D(\Omega)} C_{r}\right] \\
R_{5}(\Omega)=\frac{1}{A_{0}} \frac{1}{1+C_{i}+\alpha C_{r}}\left[\frac{\alpha}{i \Omega}+\frac{\left(\alpha\left(1+a_{i}\right)-a_{r}\right)\left(i \Omega-A_{1}\right)}{D(\Omega)} C_{r}\right]
\end{array}
\end{aligned}
$$

\section{References}

1) M. Fleming and A. Mooradian, "Fundamental line broadening of single mode GaAlAs diode lasers", Appl. Phys. Lett. 8, 511, (1981).

2) C. H. Henry, "Theory of linewidth of semiconductor lasers" IEEE QE 18, 259 (1982). K. Vahala and A. Yariv, "SemiClassical theory of noise in Semiconductor lasers “, IEEE QE 19, 1096, (1983).

3) S. Machida and Y. Yamamoto, Phys. Rev. Lett. 60, 792 (1988).

4) A. Yariv, R. Nabiev and K. Vahala, "Self quenching of fundamental phase and amplitude noise in semiconductor lasers with dispersive loss", Optics Lett. 15, 1359, (1990).

5) Y. Shevy, J. Iannelli, J. Kitching, and A. Yariv, "Self quenching of the semiconductor laser noise below the Schawlow Townes limit by using optical feedback", Optics Lett. 17, 661, (1992)

6) B. Dahmani, L. Dahmani, and R. Drullinger, Opt. Lett. 12, 876 (1987).

7) P. Laurent, A. Clairon, and C. Breant, IEEE, JQE. 25, 1131 (1989).

8) J. Iannelli, Y. Shevy, J. Kitching, and A. Yariv "Frequency stabilization and linewidth narrowing of semiconductor laser via Doppler free Faraday resonance in Cs vapor”, IEEE JQE 29, 1253, (1993).

9) J. Kitching, Y. Shevy, and A. Yariv, "1/f semiconductor laser FM noise reduction with dispersive loss", J. of Lightwave Technology 11, 1526, (1993).

10) Y. Shevy, J. Kitching, and A. Yariv, "Linewidth reduction and frequency stabilization of semiconductor laser with a combination of FM sidband locking and optical feedback", Optics Lett. 18, 1071, (1993).

11) Y. Shevy and D. Hua," Ultra Narrow and frequency stable semiconductor laser locked directly to an atomic Cs transition", Opt. Lett. 23, 472 (1998).

12) Y. Yamamoto, S. Machida, and O. Nilsson, Phys. Rev. A 34, 4025 (1986).

13) Y. Yamamoto and S. Machida, Phys. Rev. A 35, 5114 (1987), S. Machida, Y. Yamamoto and Y. Itaya, Phys rev. Lett. 
58, 1000, (1987).

14) J. Kitching, R. Boyd, A. Yariv, and Y. Shevy, "Amplitude noise reduction in semiconductor lasers using weak optical feedback with dispersive loss", Optics Lett. 19, 1331 (1994).

15) J. Kitching, A. Yariv, and Y. Shevy, "Room temperature generation of amplitude squeezed light from semiconductor laser with weak optical feedback", Phys, Rev. Lett. 74, 3372, (1995).

16) J. Kitching, A. Yariv, and Y. Shevy "Generation of amplitude squeezed light from pump suppressed semiconductor laser with dispersive optical feedback", In "Laser Frequency stabilization and noise reduction", Edited by Y. Shevy, SPIE proceedings, volume 2378, 77 (1995).

17) J. Kitching, D. Provenzano, and A. Yariv, "generation of amplitude squeezed light from a room temperature Fabry-Perot semiconductor laser”, Opt. Lett. 20, 2526 (1995). 